\title{
Zytologische Präparate
}

\section{Vorbereitung zur mikroskopischen Beurteilung}

\author{
G. Müller, I. Müller, I. Kiefer, Klinik für Kleintiere, Universität Leipzig
}

\section{Indikationen}

Die Beurteilung von Zellen zur Findung der korrekten Diagnose nimmt einen immer größeren Raum in der Kleintiermedizin ein. Insbesondere die Sonografie mit ihrer sehr hohen Sensitivität, aber nur mäßigen Spezifität, ist häufig auf die Zytologie angewiesen und in vielen Fällen stellt die zytologische Untersuchung eine Alternative zur wesentlich aufwendigeren und invasiveren Biopsie dar. Zytologische Präparate werden aber auch als „Abklatsch“, Zytobrush usw. gewonnen.

\section{Vorbereitung}

Schon bevor die Probe auf den Objektträger kommt, muss einiges beachtet werden Eine Beschriftung des Objektträgers ist unbedingt notwendig, um jederzeit eine korrekte Zuordnung zu gewährleisten und die beprobte Seite des Objektträgers zu erkennen. Deshalb sollten geschliffene Objektträger mit Mattrand verwendet werden. Die korrekte Beschriftung sollte so gewählt werden, dass auch retrospektiv eine eindeutige Zuordnung möglich ist (z. B. klinikeigene Nummer, Besitzername, Art und Lokalisation der Probe und Entnahmedatum). Sie erfolgt mit Bleistift, da alle Arten von Tinte durch die alkoholhaltigen Fixier- und Farblösungen abgespült werden.

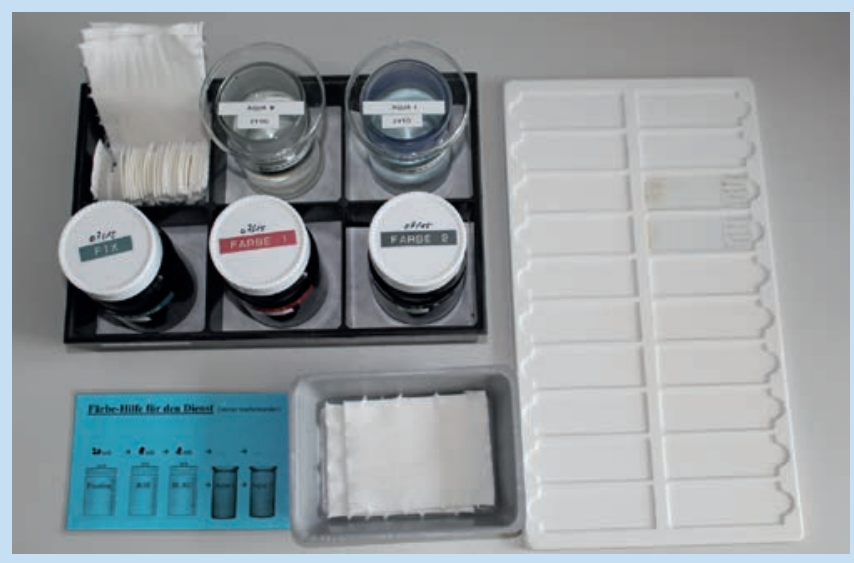

Abb. 1 Fixier- und Färbearbeitsplatz mit den beschriebenen Lösungen. Eine Kurzanleitung mit den Bearbeitungszeiten hat sich besonders bei seltenem Gebrauch oder wechselndem Personal (z. B. Nachtdienst) bewährt.

\section{Lufttrocknung}

Zum Lufttrocknen (ca. 10 min) werden die Präparate auf dafür vorgesehene Zytoständer gestellt. Wenn eine Beurteilung eilt, können die Objektträger auf der Rückseite mit einem Fön getrocknet werden, wobei die Gefahr besteht, dass eine zu große Hitzeeinwirkung die Probe unbrauchbar macht.

\section{Mikroskopische Beurteilung}

Auch wenn die Probe zur Beurteilung an einen Spezialisten gesandt wird, empfiehlt es sich zu kontrollieren, ob sich auf dem Objektträger auswertbare Zellen befinden. Das zur Beurteilung erforderliche Immersionsöl muss nach der Untersuchung wieder entfernt werden. Dafür hat sich Roti $^{\circledR}$-Histol (Fa. Carl Roth), ein Xylol-Ersatz, bewährt.

\section{Besonderheiten}

Fettige Präparate werden nach einer 24stündigen Trocknungszeit mit eisgekühltem Aceton für $1 \mathrm{~min}$ fixiert (CAVE: Unter dem Abzug arbeiten!). Danach werden sie wie oben beschrieben weiter verarbeitet.

Bei Präparaten von Lymphknoten, Milz und Leber wird ein anderes Zeitschema verwendet:

- Fixierung: $120 \mathrm{~s}$

- Farblösung I: $45 \mathrm{~s}$

- Farblösung II: 20 s

Damit wird versucht, die Färbung zu intensivieren, um die Diagnostik zu erleichtern.

Alle Abbildungen: (c) Klinik für Kleintiere, Universität Leipzig

Online zu finden unter

http://dx.doi.org/10.1055/s-0035-1558516 

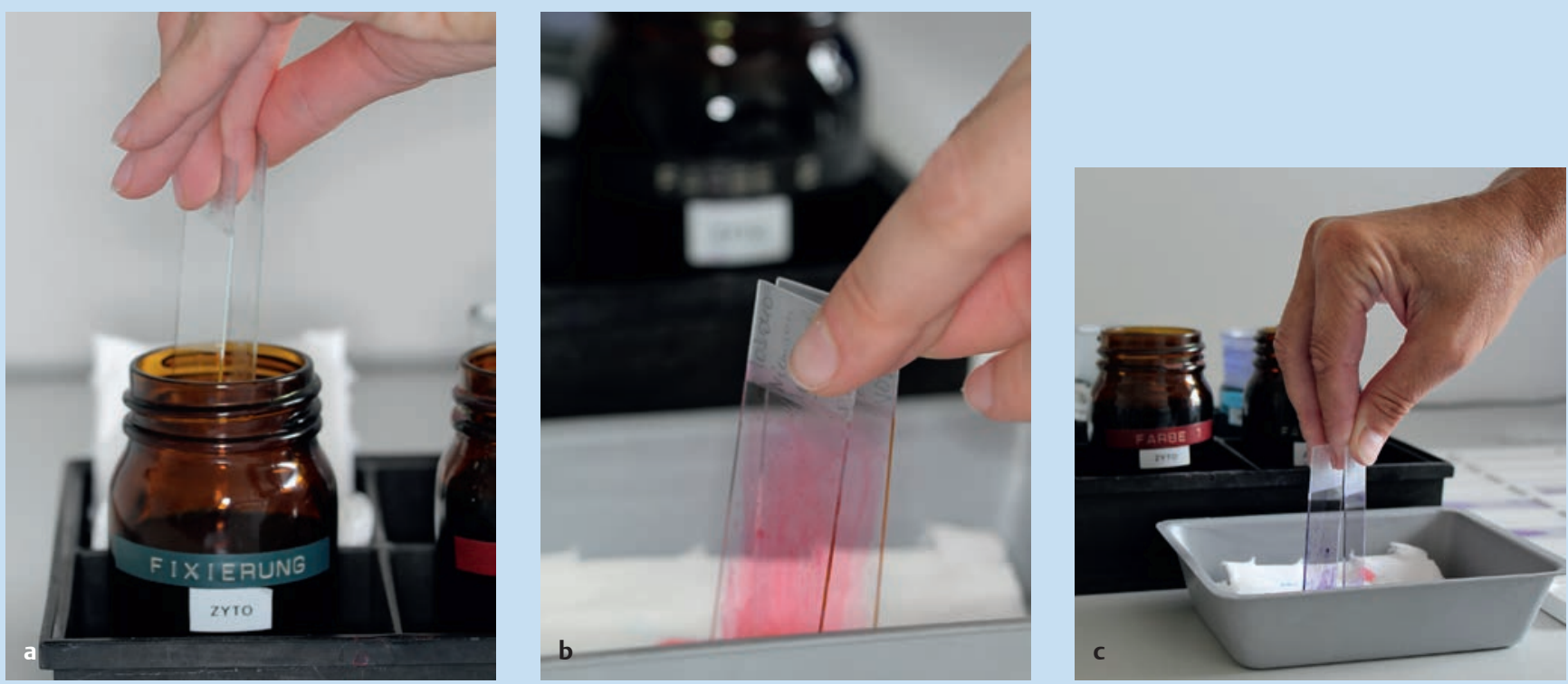

Abb. 2 Fixierung und Färbung von 2 Objektträgern gleichzeitig. Diese sollten Rücken an Rücken gehalten werden. Die Präparate werden $20 \mathrm{~s}$ fixiert und $8 \mathrm{~s}$ in jede Farblösung eingetaucht.

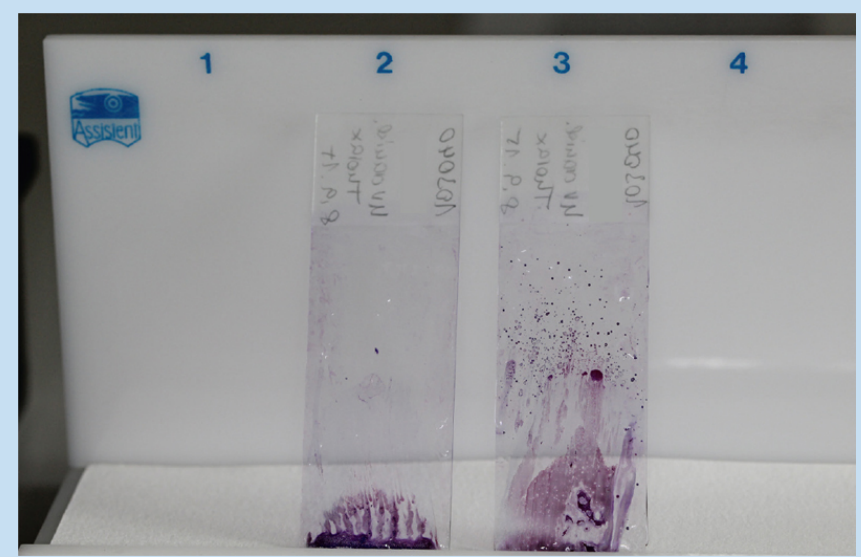

Abb. 3 Nach der Bearbeitung werden die Proben ca. 10 min luftgetrocknet.

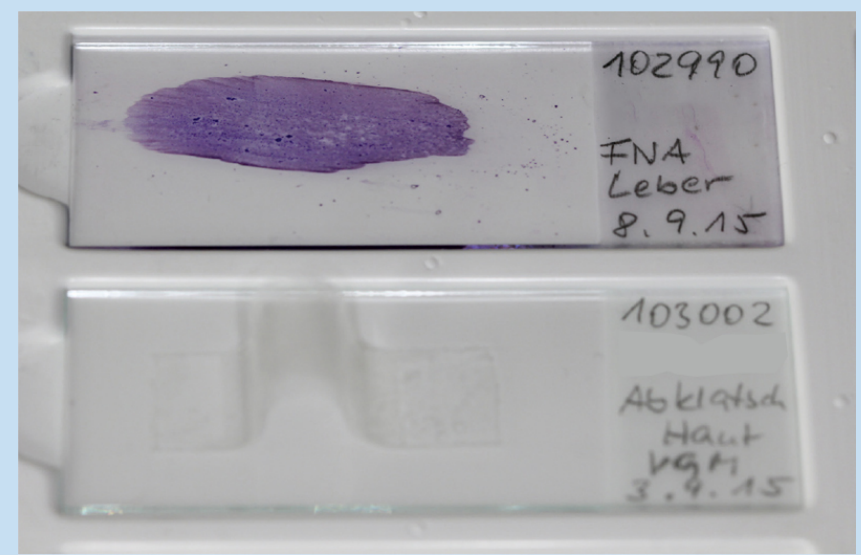

Abb. 5 Korrekt beschrifteter Objektträger mit Mattrand nach Fixierung und Färbung. Durch die Nutzung des Bleistiftes ist die Beschriftung der Objektträger auch nach der Behandlung lesbar.

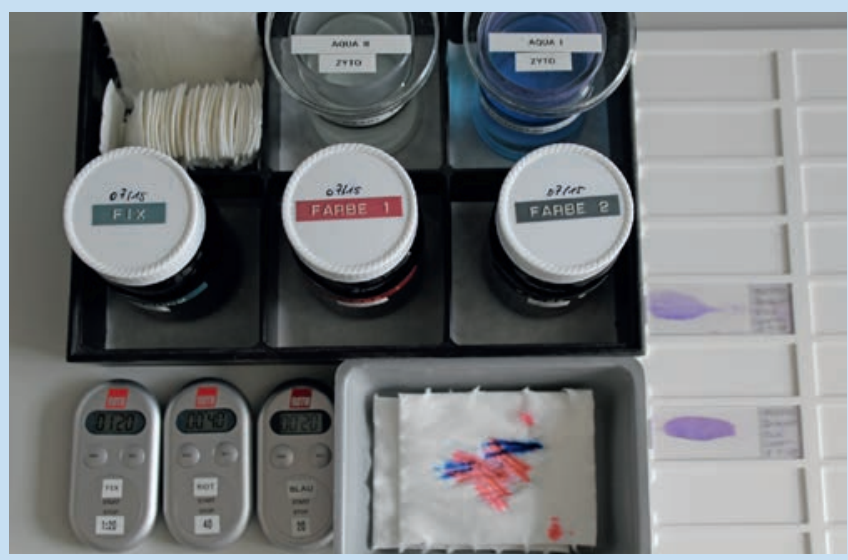

Abb. 4 Der Einsatz von Kurzzeitweckern (für jeden Arbeitsschritt einen) hat sich in der Praxis als erhebliche Arbeitserleichterung und zur Vermeidung von Fehlern als absolut vorteilhaft bewiesen.

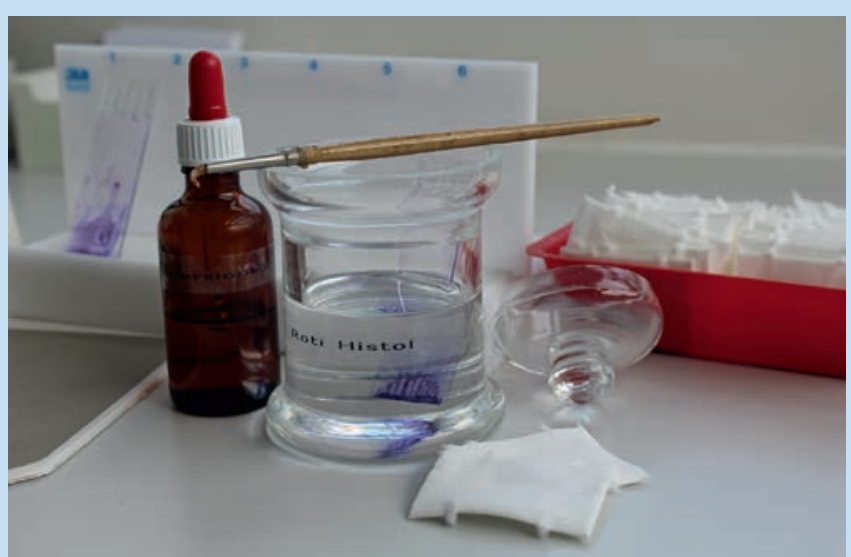

Abb. 6 Nach der Beurteilung unter dem Mikroskop wird das verwendete Immersionsöl mit Roti-Histol vom Objektträger entfernt. Die Probe kann danach archiviert oder versandt werden. 